The authors correctly point out the staggering cost of an incorrect decision which can cut both ways: It can deprive patients of effective treatments or allocation of valuable resources to ineffective drugs - resources not available for the development of novel, potentially effective drugs. The challenge to drug development is finding the best path between timely termination of ineffective drugs to save resources and full exploration of effective compounds. Futility analysis, when correctly done, do offer valuable information that can help in making this tough decision.

\section{References}

1. Aisen, P.S. and R. Raman, Futility Analyses in Alzheimer's Disease (AD) Clinical Trials: A Risky Business. J Prev Alz Dis 2020;3(7):195-196.

2. Sabbagh, M.N., et al., TTP488: From futile to fast track. Alzheimer's and Dementia, 2015. 11(7): p. P290.

\title{
Authors response to $\mathrm{D}$. Umbricht, letter to the editor referring Futility Analyses in Alzheimer's Disease (AD) Clinical Trials: A Risky Business. The Journal of Prevention of Alzheimer's Disease, 2020
}

\author{
P.S. Aisen, R. Raman \\ University of Southern California, Alzheimer's Therapeutic Research Institute, San Diego, CA \\ Corresponding Author: P.S. Aisen, University of Southern California, Alzheimer's Therapeutic Research Institute, San Diego, CA, USA, Email: paisen@usc.edu

Dear Editor,

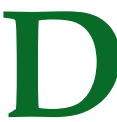
r. Umbricht suggests that the two examples we cite in our viewpoint (1) support rather than call into question the value of interim futility analyses in Alzheimer's disease (AD) trials. He points out that the first example, the Phase 3 trials of aducanumab, the futility analyses did indeed indicate a trend toward a beneficial treatment effect in one of the two trials though the planned pooled futility decision led to stopping the trials. In the second case, in which a futility analysis led to a halt, full analysis of available data suggested efficacy; a subsequent study was negative.

We certainly agree that interim data analysis can be informative. Our primary point is that interim futility analyses require assumptions and are prone to errors in design. Once futility is declared, interpretation of treatment effects is difficult and reporting evidence of efficacy is problematic. In the aducanumab case, allowing the trials to complete would likely have yielded substantially clearer evidence regarding treatment effects. Similarly, in the case of TTP488, a full dataset from a competed trial would have provided more definitive evidence one way or the other, perhaps obviating the need for a follow-up study. In both cases, the cost of a futility analysis (loss of inference from a full trial dataset) seems high, far outweighing the savings. We agree with Dr. Umbricht's call for methodologic rigor. Further, we urge that $\mathrm{AD}$ trialists carefully weigh the benefits and risks of futility analyses prior to considering them in their trial design.

\section{Reference}

1. Aisen, P.S. and R. Raman, Futility Analyses in Alzheimer's Disease (AD) Clinical Trials: A Risky Business. J Prev Alz Dis 2020;3(7):195-196. 